\section{$\underset{\substack{\text { hommes } \\ \text { \& migrations }}}{ }$}

\section{Hommes \& migrations}

Revue française de référence sur les dynamiques

migratoires

$1324 \mid 2019$

Religion et discrimination

\title{
Images confisquées, histoires invisibles
}

\section{Marianne Amar}

\section{(2) OpenEdition \\ Journals}

\section{Édition électronique}

URL : https://journals.openedition.org/hommesmigrations/8811

DOI : 10.4000/hommesmigrations.8811

ISSN : 2262-3353

\section{Éditeur}

Musée national de l'histoire de l'immigration

\section{Édition imprimée}

Date de publication : 1 janvier 2019

Pagination : 140-146

ISBN : 978-2-919040-44-5

ISSN : $1142-852 X$

\section{Référence électronique}

Marianne Amar, «Images confisquées, histoires invisibles », Hommes \& migrations [En ligne], 1324 |

2019, mis en ligne le 01 janvier 2022, consulté le 07 janvier 2022. URL : http://

journals.openedition.org/hommesmigrations/8811; DOI : https://doi.org/10.4000/

hommesmigrations.8811 


\title{
RECHEDERCHE
}

\section{Images confisquées, histoires invisibles}

\author{
Marianne Amar, \\ cheffe du département Recherche, Musée national de l'histoire de l'immigration.
}

Les musées de migration sont nés d'un paradoxe. Leur revient en effet de rendre visible la place des migrants dans l'histoire du pays afin de contribuer à leur reconnaissance dans l'espace public. Or comment rendre compte, dans l'exposition, d'une histoire justement marquée par l'invisibilité sociale? Avec quelles images, quelles traces laissées, faire malgré tout récit du passé ? Comment ne pas recouvrir le manque d'image par des images de substitution - notamment contemporaines - que l'on exposerait «faute de mieux ", pour conjurer la peur du vide, au lieu d'en faire l'un des enjeux de l'exposition? Exposer, enfin, suffit-il à rendre visible et à reconnaître? « Je suis invisible, comprenez bien, parce que les gens refusent de me voir \#, constatait ainsi le héros de Ralph Ellison'.

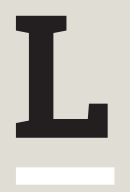

a rencontre organisée par le Musée national de l'histoire de l'immigration aux derniers Rendez-vous de l'histoire de Blois², intitulée « Les images confisquées. Comment exposer des histoires invisibles? », proposait de déplier ce paradoxe, en confrontant les migrations à d'autres histoires marquées par ces enjeux d'invisibilité : les destructions et les spoliations d'œuvres pendant la Seconde Guerre mondiale avec Arno Gisinger ${ }^{3}$; la photographie des mondes tsiganes avec Ilsen About ${ }^{4}$; la présence des femmes et des hommes noirs dans la création picturale avec Anne Lafont ${ }^{5}$. À travers cette énumération, on mesure combien le terme d'« invisibilité » peut recouvrir des réalités différentes, prises dans une chronologie large. Il renvoie à tout ce qui ne peut être vu: images absentes d'une réalité que l'on n'a pas voulu saisir ou qui s'est dérobée ; images détruites, objets volés, confisqués; mais aussi trop-plein d'images qui empêche de voir, stéréotypes

1. Ralph Ellison, Homme invisible, pour qui chantes-tu?, Paris, Grasset, 2002.

2. Consacrées cette année à «La puissance des images ».

3. Photographe et maître de conférences à l'université Paris 8.

4. Chargé de recherche au CNRS, Centre Georg Simmel, et commissaire de l'exposition « Mondes tsiganes. Une histoire photographique, 1860-1980», Musée national de l'histoire de l'immigration, 13 mars-26 août 2018.

5. Directrice d'étude à l'EHESS. 
et images qui «mentent», détournées de leur sens par la légende ou les processus d'exposition. Mais toutes posent au musée la même question : comment dénouer ces processus d'invisibilité, mais aussi les rendre sensibles dans le musée?

\section{Spoliations nazies, disparition des œuvres et traces photographiques}

Dans cette réflexion, la spoliation des populations juives en Allemagne, en Autriche, puis dans toute l'Europe occupée porte une forme de contradiction, dont témoigne le travail d'Arno Gisinger. Car le régime nazi voulait tout à la fois faire disparaître de l'espace public les œuvres pillées, voler et dérober à la vue de leurs propriétaires légitimes les biens ordinaires, mais aussi témoigner que ce travail avait été mené avec efficacité, et donc l'enregistrer et le photographier pour en conserver une trace. Depuis la guerre, les travaux sur les spoliations ont utilisé ces images fabriquées par les spoliateurs, mais en

(2) C'est en artiste qu'Arno Gisinger traite des manières de pallier l'absence des œuvres disparues dans le processus de spoliation. ont retourné l'usage. Ce sont elles, en effet, qui ont permis de commencer l'enquête pour retrouver " physiquement » les œuvres et les biens volés. De cette articulation entre spoliation et photographie comme preuve et trace, Arno Gisinger cite deux exemples. D'abord, le travail de Sarah Gensburger autour de l'album photographique retrouvé du pillage des biens juifs à Paris entre 1940 et $1944^{6}$. Second exemple : les photographies prises au musée du Jeu de Paume à Paris, dans la « salle des martyrs » où étaient rassemblées les œuvres d'art moderne, volées à leurs propriétaires juifs, avant d'être revendues, échangées ou acheminées en Allemagne. Là encore, les enjeux de visibilité/invisibilité s'emboîtent les uns dans les autres: les œuvres spoliées sont dérobées à la vue, interdites d'exposition, cachées dans une salle du Jeu de Paume protégée par un grand rideau noir, mais néanmoins admirées par quelques visiteurs privilégiés, à commencer par Hermann Goering qui venait y puiser pour sa collection personnelle, et surtout photographiées de manière systématique par l'occupant, ce qui a aidé le patient travail de récupération - toujours inachevé - après la guerre ${ }^{7}$.

C'est en artiste qu'Arno Gisinger traite des manières de pallier l'absence des œuvres disparues dans le processus de spoliation. De ses travaux présentés à Blois, on retiendra celui mené autour du tableau d'Otto Dix « La veuve ». Cette œuvre figurait parmi les nombreux tableaux d'art moderne et contemporain acquis par la Kunsthalle de Mannheim après la Première Guerre mondiale. Elle

6. Sarah Gensburger, Images d'un pillage. Album de la spoliation des Juifs à Paris, 1940-1944, Paris, Textuel, 2010.

7. Sur les spoliations d'œuvres pendant la Seconde Guerre mondiale, on consultera parmi les ouvrages en français : Corinne Bouchoux, Laurent Douzou, « Si les tableaux pouvaient parler... ». Le traitement politique et médiatique des retours d'œuvres d'art pillées et spoliées par les nazis (France 1945-2008), Rennes, Presses universitaires de Rennes, 2013; Hector Feliciano, Le musée disparu. Enquête sur le pillage d'œuvres d'art en France par les nazis, Paris, Gallimard, 2003 ; Les Archives diplomatiques, Jean-Marc Dreyfus, Le catalogue de Goering, Paris, Flammarion, 2015. 
sera accrochée en 1925 dans la grande exposition du Musée intitulée « Nouvelle objectivité 》, puis exposée en 1933 à l'occasion de la manifestation « Kulturbolschewistische Bilder ». Entre-temps, le contexte a radicalement changé. Les nazis sont désormais au pouvoir et le musée, dans le droit-fil des nouveaux impératifs culturels, entend dénoncer la politique d'acquisition menée sous Weimar et sa prédilection affichée pour « l'art dégénéré ». Les tableaux exposés en 1933 vont en partie disparaître dans les spoliations : certains seront retrouvés après 1945, d'autres non, et « La veuve » d'Otto Dix a sans doute disparu à jamais. Pour rendre sensible son absence, Arno Gisinger a travaillé sur ce qui reste: un ensemble de plaques de verre, œuvre d'un photographe qui avait méthodiquement documenté la collection de la Kunsthalle, et les archives de l'institution. Dans un dispositif complexe, installé dans le château d'eau situé à proximité de l'ancien musée et ouvert au public pour l'occasion, il a fait revivre les traces de ces tableaux disparus, en projetant en très grand format leur empreinte laissée sur les plaques de verre. «La veuve» d'Otto Dix a ainsi pu être à nouveau " exposée », avec un commentaire de Gisinger en voix off. L'archive documentaire - la plaque de verre - revisitée par le geste de l'artiste contemporain permet ainsi de suppléer à l'absence de l'œuvre elle-même. Après la guerre, le directeur du musée avait imaginé tout autre chose. Il avait acquis auprès d'Otto Dix, un autre tableau, « La folle». Point commun avec «La veuve» : c'est le même modèle qui posait. Pour le directeur, ce geste de muséologie permettait à sa manière de remplacer le tableau manquant.

\section{Quand le musée rend invisible : la représentation des Noirs au XVIII' siècle}

Anne Lafont fait un retour vers un passé plus lointain puisqu'elle aborde la représentation des femmes et des hommes noirs dans la peinture du XVIII siècle. Ce qui l'intéresse ici, explique-t-elle, c'est de regarder ces peintures avec attention, au-delà de la description littérale de l'image, "par-delà l'intention déclarée de l'auteur ou de l'idéologie supposée du contexte ». Ce qui se révèle alors, c'est en fait l'hétérogénéité des situations, leur complexité, même dans des relations de domination et d'asymétrie a priori rigides. Ainsi, un dessin de Carmontelle (1717-1806) représentant Narcisse, homme noir attaché à la cour 
du duc d'Orléans, assis aux pieds d'une femme au clavecin. La lecture attentive de l'œuvre met en lumière un réel qui nuance, voire contredit, ce que l'on attend. La condition servile du personnage se devine à travers le collier - collier d'esclave - porté par Narcisse. Mais le soin apporté aux vêtements, l'apparence, la pose, une certaine aisance en recouvrent la portée et laissent au contraire affleurer une forme de relation presque à parts égales. Dans cette scène au clavecin, Carmontelle montre aussi un savoir élitiste partagé, celui du solfège et de la musique écrite, qui construit une communauté de savoirs qui va à l'encontre des préjugés. Dans le musée, la gestion des œuvres peut aussi construire de l'invisibilité, à travers plusieurs opérations successives : modalités de l'inventaire, récolement, choix de l'exposition ou de la mise en réserve, dicibilité de l'œuvre via le cartel. «Ces gestes techniques, professionnels, posés suivant un savoir manuel et intellectuel, ne sont jamais anodins, neutres ou transparents 》, souligne Anne Lafont. Ils portent « une succession de choix issus d'une formation professionnelle, qui peuvent favoriser une forme de dissimulation ou d'invisibilisation dans le tableau ». L'histoire de l'œuvre ne peut donc être détachée de son histoire dans l'institution, de sa transmission dans le temps long, des silences et des tabous qui ont pu l'accompagner. Témoin, une œuvre d'Antoine Coypel, exposée au Louvre et datée de 1684. C'est l'une des premières œuvres sur laquelle apparaît un page noir dans les arts visuels français et l'on dispose de l'historique complet de sa fortune critique dans les collections royales, puis dans les musées. Jusqu'à récemment, ce tableau portait pour titre « Jeune fille caressant un chien ». Et puis l'intitulé a changé dans les années 2000 : Le Louvre l'intitule désormais « Jeune noir tenant

(2) L'histoire de l'œuvre ne peut donc être détachée de son histoire dans l'institution, de sa transmission dans le temps long, des silences et des tabous qui ont pu l'accompagner. une corbeille de fruits et Jeune fille caressant un chien ». Le titre choisi au $\mathrm{XX}^{\mathrm{e}}$ siècle, qui euphémise la réalité et sa représentation visuelle, tranche avec les inventaires et les catalogues du XVIII ${ }^{e}$ siècle qui avaient au contraire essayé d'intégrer la complexité de ce petit tableau. Ainsi, un inventaire de 1709 évoque la peinture en ces termes: «Une femme qui pose sa main sur un petit chien et un Maure sur le devant qui tient un panier de fruits qu'un singe embrasse $\gg$. Et puis en 1733, l'inventaire de Meudon précise que le tableau représente « une petite femme, vêtue de bleu, tenant dans ses bras une petite barbette et un nègre avec deux plumes rouges, tenant une corbeille de fruits, un tapis d'étoffe d'or, doublé de vair avec frange d'or ». Et de surcroît, en marge, on peut lire que le jeune noir était « Angola, nègre trompette, du feu Roi avec sa maîtresse ». En marge, figurent aussi une série d'informations sur le modèle - il aurait, suggèrent les archives, été en relation sentimentale, voire sexuelle, avec cette jeune femme en bleu - qui ont été ignorées par la conservation à partir du XIX ${ }^{\mathrm{e}}$ siècle. Comme si, dans le processus d'institutionnalisation des musées, ces informations ne pouvaient plus être prises en charge. Ce qui a conduit, via les cartels et le titre de l'œuvre, à rendre invisible une partie de sa signification. 


\section{Contre les stéréotypes : une exposition des " Mondes tsiganes »}

Troisième terrain d'étude présenté par Ilsen About: la représentation photographique des populations appelées «tsiganes », qui a fait l'objet d'une exposition au Musée national de l'histoire de l'immigration ${ }^{8}$. Son enjeu, et celui du travail scientifique qui l'a précédée, était de «saisir les traces photographiques d'une population décrite par l'image comme un peuple homogène, isolé dans l'espace et l'histoire, présent à la fois partout et nulle part », explique Ilsen About. Ici, l'invisibilité ne se construit pas sur l'absence ou sur les manières de nommer, mais sur la surabondance d'images stéréotypées qui alimentent un imaginaire occidental et masquent l'histoire, située dans le temps et dans l'espace, des individus, des groupes, des familles. En témoignent, par exemple, les nombreux magazines exposés dans l'exposition qui répètent à l'infini les mêmes images, sous les mêmes titres, chargés de susciter peur et rejet en évoquant le crime ou le vol.

Pour rompre avec cette forme d'invisibilité, l'exposition a voulu mener un double travail : d'abord, déconstruire les stéréotypes, mais aussi révéler, presque au sens photographique du terme, l'histoire sociale de ces populations à travers une relecture des images connues et la mise à disposition du public de fonds inédits. S'agissant des stéréotypes, le devenir d'un reportage de Denise Bellon dans la « zone » à Paris, en 1938, permet de mieux comprendre comment ils se construisent et se renforcent. Cinq ans plus tard en effet, une des photographies - le mariage entre une Gitane et un Sinto sud-africain - se retrouve sur la couverture du magazine Regards pour illustrer les persécutions dont ont été victimes les Tsiganes en Europe pendant la Seconde Guerre mondiale. Ce mésusage de l'image, arrachée à son contexte de production, s'inscrit dans une réalité plus large. Le stock de photographies disponibles concernant ces populations apparaît en effet si réduit, limité à quelques figures de style, qu'elles sont répétées à l'infini, de publication en publication, jusqu'à les figer dans des lieux communs visuels en dépit de la diversité des situations historiques.

8. L'exposition est le fruit du travail collectif de trois commissaires : Ilsen About, Mathieu Pernot et Adèle Sutre. On pourra se référer au catalogue qu'ils ont codirigé : Mondes tsiganes. Une histoire photographique, 1860-1980, Paris, Musée national de l'histoire de l'immigration/Actes Sud, 2018. 
Pour renverser la force des stéréotypes et essayer de contribuer, par l'image, à l'histoire de ces populations, l'exposition s'est d'abord attachée à incarner les femmes et les hommes qui figuraient sur l'image. D'abord en retrouvant les modèles : qui sont ces personnes qui posent devant le photographe? Ont-elles tenu un rôle dans la construction de la représentation? Comment, surtout, « retrouver leur identité véritable derrière ce fleuve d'images qui les recouvre et nous recouvre, en effaçant leur singularité et leur diversité » ? À cet égard, les différentes enquêtes menées en Grande-Bretagne dans les années 1910 autour d'un groupe itinérant apparaissent exemplaires, puisque l'on peut, en les croisant, mettre en lumière la diversité sociale et familiale du groupe, et surtout nommer chacun des membres de la famille. Le travail du Père Joseph Valet (né en 1927), installé à Clermont-Ferrand dans une grande proximité avec les familles manouches d'Auvergne et du Massif central, s'inscrit dans la même démarche. Ses images révèlent à la fois les conditions difficiles de l'enquête, mais aussi un jeu - au sens

(ब Certaines images nomment bien les hommes et les femmes qu'elles saisissent dans le cadre, sans pour autant échapper aux canons de la photographie typique, ethnographique, qui isole les personnages dans une fonction professionnelle. ludique du terme - à l'œuvre entre le photographe et ses modèles. L'enquête, menée dans ces conditions, peut révéler des manières de faire, d'habiter dans des sociétés qui sont parfois difficilement accessibles par d'autres sources que la photographie. On est loin de cette autre image montrée dans l'exposition: une carte postale diffusée massivement au début du $\mathrm{XX}^{\mathrm{e}}$ siècle qui désigne un groupe sous le terme de «tribu de Romanichels ». Reste que nommer ceux qui sont photographiés, donc les incarner, apparaît comme une condition essentielle de la déconstruction des stéréotypes - «le Tsigane » n'existe pas - mais insuffisante. Certaines images nomment bien les hommes et les femmes qu'elles saisissent dans le cadre, sans pour autant échapper aux canons de la photographie typique, ethnographique, qui isole les personnages dans une fonction professionnelle.

En fait, il n'est jamais simple d'exposer une histoire, non pas trouée par le manque d'images, mais sans cesse confrontée aux stéréotypes. Comment par exemple, s'interroge Ilsen About, rendre compte des dispositifs mis en œuvre par l'État pour contrôler les populations dites «nomades » à partir de 1912, tout en rendant hommage aux personnes visées par ces procédés? Les commissaires de l'exposition ont choisi de rendre compte, sur un même panneau, des formes prises par le contrôle et de la diversité des visages. Ainsi, sur le mur principal de l'exposition, figuraient des reproductions de plaques de verre issues du fonds photographique de la Sûreté ${ }^{9}$ et jamais montrées à ce jour. S'y trouvent saisies une série inédite de photographies de «nomades 》, « romanichels 》, « bohémiens » - comme on les nommait alors - qui datent des années 1907-1908. Par-delà leur origine policière, elles dressent ensemble un portrait collectif placé sous le 
signe de la diversité. Diversité des visages et des âges, mais aussi des communautés tsiganes en France saisie à travers les noms : Sintis aux noms à consonance germanique comme les Reinhardt, ou italienne pour ceux qu'on appelle Piémontais ; Roms balkaniques ou russes, Manouches, ou encore Gitans.

En fin de compte, l'exposition a réussi à tenir trois fils : montrer des images inédites ; produire, à travers l'image, de nouveaux savoirs ; saisir l'interaction entre le photographe et ses modèles. Cet « événement» de la photographie, pour reprendre le terme de Susan Sontag, l'image en est à la fois l'occasion et la preuve, puisqu'elle le provoque et en rend compte dans le même mouvement. $\mathrm{Au}$-delà des mondes tsiganes, ce travail sur les conditions de production de l'image apparaît partout comme une des conditions de la visibilité. Pour faire retour sur d'autres époques et d'autres images montrées dans les travaux d'Arno Gisinger et Anne Lafont, on y ajoutera l'indispensable réflexion sur les modalités d'exposition, de documentation, et donc de réception des œuvres. Privée de mots, et de mots justes, l'image reste en partie invisible. 